\title{
Probabilistic sensor design for healthcare technology
}

\author{
Joseph Russell \\ Department of Engineering Science \\ University of Oxford \\ Oxford, United Kingdom \\ joseph.russell@worc.ox.ac.uk
}

\author{
Jeroen Bergmann \\ Department of Engineering Science \\ University of Oxford \\ Oxford, United Kingdom \\ jeroen.bergmann@eng.ox.ac.uk
}

\begin{abstract}
In healthcare technology, failure to accurately detect a medical event, such as electromyographic (EMG) activity or the onset of a heart attack, could have serious consequences for the user. The application of sensor networks to optimize event detection is therefore a key area of biomedical engineering research. The optimum number of sensors for event detection can be difficult and time-consuming to determine experimentally. A novel probabilistic model could be used to estimate the appropriate number of sensors required and is described in this paper. A simple statistically independent model (naïve approach) is introduced, which may be useful but relies on a set of assumptions that do not reflect most real-world applications. From there, a more practical, revised model that can be more easily applied to real-life systems was developed. Both models were subsequently tested against experimental data from a real EMG system. While the naïve approach was practically unachievable, the model was found to be mathematically sound. Predictions from the models were compared and the revised model was found to be more accurate than the naïve model. The revised model was verified against a majority voting system and it successfully predicted sensor network performance with only a small margin of error. This work presents a probabilistic sensor network approach to virtually exploring possible networks and informing optimum design of sensor networks used in healthcare and industry.
\end{abstract}

\section{INTRODUCTION}

Research into body area networks has become increasingly important in recent years, with rising medical costs and an ageing world population motivating the development of cheaper, more efficient technologies to assist in the care of patients with a wide variety of conditions [1]. One such area is artificial limbs, where control interface technology such as electromyography (EMG) depends on the use of accurate, reliable sensor information from an inherently noisy, unreliable source [2]. EMG is the practice of monitoring the electrical activity in a set of muscles and is widely used in medical applications such as prosthetics.

The use of sensor networks to combine results from multiple sensors into a single, more reliable measurement could be of great benefit in EMG and other wearable technology applications [3]. Establishing a framework to model a sensor network's performance, efficiently combine its sensor information, and predict the optimum number of sensors would be particularly useful in sensor network design, enabling virtual prototyping [4] and reducing the need for extensive experimentation. The report will explore a "naïve" model that does not take into account false positives and negatives and proposes a "revised" model that does. An error between the experimentally determined values and those of the models can be calculated to capture model performance.

The aim of this paper is to determine the root mean square error (RMSE), absolute and mean error between the measured and predicted probabilities across different models. The event detected will consist of the activation of the lower-arm muscles (active when closing the hand), which is an event often used in EMG-based trans-radial prosthetic control. The procedures developed will be kept as broad as possible to allow for generalisation to other event-detecting scenarios.

\section{REVIEW OF EXISTING RESEARCH AND LITERATURE}

A sensor network is a system of multiple sensors cooperating to sense physical phenomena [5]. Before such a system can be designed, a model can be produced to mathematically analyse the probabilities of detecting the event. The simplest model would be a naïve model that assumes statistical independence [6].
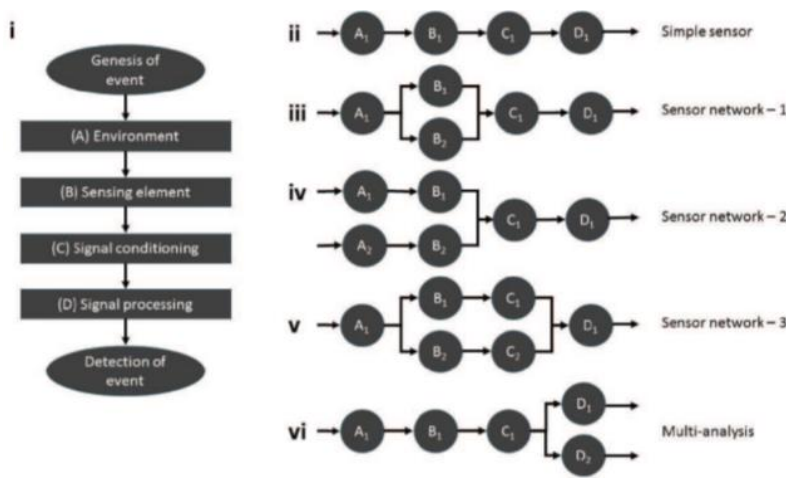

Fig. 1. Model for event detection (taken from [6]). (i) shows the steps that lead up to the detection of a given event. (ii) shows the most simple design. (iii) displays a network design that consist of two sensors (iv) this is a network that has sensors that sense different "parts" of the environment. (v) shows a network containing two different sensors, which require different signal conditioning (vi) shows a sensing configuration that is processed in two different ways.

Fig. 1 shows a diagram of the (naïve) model, demonstrating how the detection event is broken down into different stages. Each stage is assigned its own probability, indicating the chance that a signal will successfully pass through the stage without being lost. A signal must successfully pass through all four stages in order to register as detected. Fig. 1 also shows examples of how the network can be modified - by sensing from multiple environments, adding extra sensors, using multiple conditioning methods, or combining decisions from multiple processing methods.

Assuming independence between stages, the probability of event detection $(P)$ for a design consisting of a single sensor node (Fig. 1.ii), where $P(A 1), P(B 1), P(C l)$ and $P(D 1)$ are the probabilities associated with each stage, is given by:

$$
P(A 1 \cap B 1 \cap C 1 \cap D 1)=P(A 1) \cdot P(B 1) \cdot P(C 1) \cdot P(D 1)
$$


The probability will change depending on how the design affects the different stages.

\section{Stage (A) - Environment}

The first stage of the sensor network detection process is the environment stage. This is the probability that, when an event occurs, a measurable signal identifiable as the event is produced in the region being sensed. The choice of sensing environment is very important in any sensor network.

In EMG, each muscle being sensed might be considered to be its own environment. The placement of the sensors in the environment will influence the probability of correctly detecting an event, such as muscle activation [7]. Furthermore, if these muscle sites are close to one another, there may be the possibility of cross-talk [8].

\section{Stage (B) - Sensing Element}

The second stage of the process is the sensing element. This is the probability that the sensor used is capable of picking up the signal from its environment. The naïve model assigns a single probability to the proportion of correctly identified events (the sensitivity), but does not consider the possibility of false positives (the specificity). These are pliable in relation to each other, as it is impossible to determine specificity merely from information about the sensitivity [9].

Typically, in the context of EMG, if the sensor is chosen well, this sensitivity probability is very high $(\sim 1)$. Choosing a sensor that has insufficient precision or accuracy will produce a lower probability.

\section{Stage $(C)$ - Signal Conditioning}

The third stage of the process is signal conditioning, which can consist of filtering or amplification [10]. It can be described as the probability of an event passing through any conditioning, such as noise filtering, which takes place.

However, this stage should also encompass the probability that not enough filtering has been done. For instance, a system might detect a noisy signal perfectly from the environment in stage A with a perfect sensor in stage B, but if no filtering takes place, Stage $\mathrm{C}$ might still output an imperfect probability of detection. Adding an appropriate filter (such as a notch filter for single-frequency noise) could completely remove the noise, increasing the Stage C probability to almost 1 . As such, a more precise definition of the signal conditioning probability would be the probability that a signal containing the event information, once passed through any conditioning steps, is still measurable and not corrupted by noise.

\section{Stage (D) - Signal Processing}

This final stage, signal processing, may be defined as the manipulation of random signals to extract pertinent information about the underlying mechanisms that generate or transform them [11]. It covers any operations performed using the signal after conditioning, be that comparison to a threshold value, pattern recognition, etc. It is the probability that the software correctly detects that an event has occurred, using the signal that has passed through the other stages. For a sensor network, any fusion of signals can take place in this stage.

\section{MATHEMATICAL MODELLING}

\section{A. Accounting for dependency}

The naïve model assumes the sensor data readings to be independent. This assumption does not hold in most realworld scenarios. For two sensors acting together, with individual probabilities of $\mathrm{P}(\mathrm{B} 1)$ and $\mathrm{P}(\mathrm{B} 2)$, the total probability of the sensor stage, $\mathrm{P}(\mathrm{B})$, can be given by:

$$
P(B)=P(B 1 \cup B 2)=P(B 1)+P(B 2)-P(B 1 \cap B 2)
$$

This may be rewritten [12] as:

$$
P(B 1 \cup B 2)=P(B 1)+P(B 2)-P(B 1 \mid B 2) \cdot P(B 2)
$$

The sensors may be dependent on one another, due to, for example, crosstalk. Commonly, this will be a positive dependency, i.e. when one sensor is high, the other is also likely to be higher. As such, it can be expected that:

$$
P(B 1 \mid B 2) \geqslant P(B 1)
$$

Therefore, the combined sensor probability predicted by the naïve model will be an overestimate and provides an upper bound for the true value - the magnitude of the difference will depend on the degree of dependency.

If the sensors are totally dependent on one another - i.e. B1 is only 1 if B2 is also 1 - adding sensors will not increase the combined sensor network probability.

\section{B. Accounting for the Confusion Matrix}

1) The Importance of Accounting for All Four Quadrants

The naïve model considers only the top-left quadrant of the confusion matrix [13], assuming that if any of the sensors in the network detects an event, then it is a true positive.

In an EMG system, however, false positives are just as much of a consideration as true positives. The noisy nature of EMG signals means, in order to achieve required sensitivity levels, the specificity must consequentially suffer. The choice of activation threshold is a trade-off between these two factors, which are equally important.

\section{2) Combining sensor readings}

One established method of combining sensor readings is majority voting, which simply counts how many sensors support each possible outcome and selects the result supported by the most sensors. This technique is readily applicable to a range of scenarios [14], including EMG, and so a majority voting system will now be discussed in detail.

The majority voting system used for this application will have two possible outcomes: either the event is occurring, or it is not. Therefore, at least half of the sensors must agree on the outcome. If equal numbers of sensors detect and do not detect an event, an arbitration system will be employed.

For the purpose of simplification and clarity, it will be assumed that sensors used have the same specifications, and that the sensitivity and specificity of the sensors are the same.

Majority voting is most effective when the first assumption holds true, and if it does, it is possible to establish a mathematical rule predicting the network's performance. This model will therefore incorporate this assumption, and a more effective technique for situations where this assumption is not true will be considered at a later stage.

The probability $P(S)$ that an individual sensor correctly detected whether or not the event has occurred, is assumed to simply be equal to both sensitivity and specificity. 
The probability $P(B)$, of the combined sensor network correctly detecting whether or not an event has occurred, varies depending on the number of sensors $(n)$. For a single sensor, it is obvious that the probability of the sensor network choosing a value that is correct is equal to the probability of the individual sensor correctly detecting a signal, i.e.

$$
P(B)=P(S)
$$

For two sensors or more an arbitration system is used.

\section{3) A Revised Model}

Applying these principles to larger numbers of sensors shows that for a number of sensors $n$, where $n$ is even, the sensor network has the same probability of being correct as a system of $n-1$ sensors. For example, the resultant probability of a sensor network being correct with 3 sensors is

$$
P(B)=P(S)^{3}+3 P(S)^{2} \cdot(1-P(S))
$$

For 5 sensors we get,

$$
P(B)=P(S)^{5}+5 P(S)^{4} \cdot(1-P(S))+10 P(S)^{3} \cdot(1-P(S))^{2}
$$

and 7 sensors yields

$$
\begin{gathered}
P(B)=P(S)^{7}+7 P(S)^{6} \cdot(1-P(S))+21 P(S)^{5} \cdot(1-P(S))^{2}+ \\
35 P(S)^{4} \cdot(1-P(S))^{3}
\end{gathered}
$$

These equations demonstrate a clear pattern, which can be summarized into a final equation as follows,

$$
\begin{gathered}
P(B)= \\
\left\{\begin{array}{c}
\sum_{k=0}^{\frac{n-1}{2}} C_{k} \cdot(P(S))^{n-k} \cdot(1-P(S))^{k} \\
\sum_{k=0}^{\frac{n}{2}-1}{ }_{n-1} C_{k} \cdot(P(S))^{n-1-k} \cdot(1-P(S))^{k}
\end{array}\right.
\end{gathered}
$$

if $n$ is odd

if $n$ is even

where $n$ is the number of sensors, $k$ is a dummy variable for summation and ${ }_{n} C_{k}$ is the binomial coefficient. In a reallife situation, where sensors can be wrong, this model gives a much more accurate estimate of the sensor network's performance than the naïve model.

\section{METHODS}

\section{A. EMG Hardware and Software}

The EMG sensor kit used was the BITalino (BITalino Freestyle, PLUX Wireless Biosignals, Lisbon, Portugal), a low-cost body-sensing board. It is able to sense up to five EMG inputs at a time - enough to test the sensor network system in action. Each input is connected to two sensing electrode pads placed on the muscle and a ground reference pad, placed on the collarbone. It runs at a sample rate of $1000 \mathrm{~Hz}$ [15]. Data was recorded using OpenSignals (r)evolution (Public Build 2018-12-04, PLUX Wireless Biosignals, Lisbon, Portugal). All subsequent processing was done in MATLAB (R2015b, Mathworks Inc, Natick, MA, USA).

\section{1) Designing an Experiment}

To test the model, a method was needed for producing EMG signals under controlled, repeatable conditions, alongside an absolute ground truth indicating whether or not the event was occurring.

The action chosen for detection was the periodic activation of muscles that close the hand. The rest of the arm was allowed to rest on a desk, being kept entirely still. Surface electrodes were placed on five different major muscle sites - the extensor digitorum, the flexor carpi radialis, the triceps, the biceps and the lower deltoids, following standard placement recommendations provided by SENIAM [16]. Maximum activity would be expected in the flexor and extensor muscles, with the magnitude (and therefore accuracy) expected to decrease with distance from the hand.

To create a ground truth, a simple online metronome [17] was used. Set to produce an audible beep at a rate of $0.5 \mathrm{~Hz}$, this allowed the test subject to open and close their hand at regular two second intervals. Every time the metronome "ticked" when their hand was open, the subject closed their hand such that the springs of the finger exerciser were fully compressed. This position was then held until the metronome "ticked" again, when they fully relaxed their hand. This was repeated for three minutes, or until the subject was no longer able to fully compress the springs.

The data obtained from this experiment was used to identify the probabilities of each stage of the naïve model and compare the accuracy of the combined system with the model's predictions. This was done by constraining the experiment's conditions to set the probability of each stage to $\sim 1$, and then relaxing the constraints one by one and measuring the resultant probabilities, which were assumed to be approximately equal to the product of the four stages.

\section{B. EMG Sensor Processing}

Data from the BITalino is imported into MATLAB, and artificial noise is added using a pseudorandom sequence, with tunable magnitude. The data is was processed using standard EMG methods [18]. An optimal voltage threshold is selected and when the smoothed signal is above or equal to this threshold, it is assigned the value of "1", anything below is " 0 ".

\section{1) Sensor Fusion}

There are up to five data channels originating five sensors, each individually filtered and processed (using general techniques) into $1 \mathrm{~s}$ and $0 \mathrm{~s}$. The data is combined using three methods that are described below.

\section{a) Näve Model Fusion}

The naïve model assumes that if any of the sensors detects an event, then the event has happened, with no possibility of error. To recreate this situation in the EMG example, each of the sensors in the network is compared to the ground truth. If any of their measurements is found to be correct, then the correct value is used as the network output. If not (i.e. they all agree on an incorrect value), then that value is used instead.

This technique forms an upper bound on the accuracy of the sensors - the best theoretical result the network of sensors could possibly have achieved, as only one sensor needs to be correct for the system to be correct.

\section{b) Majority Voting}

As previously discussed, majority voting is a straightforward, well-established method of combining multiple readings for any discrete "multiple-choice" system. 
One of the downsides of majority voting is that it does not account for differences in accuracy between measurements. A badly placed sensor which is only accurate $60 \%$ of the time makes the same contribution as an excellent sensor with a 99.9\% accuracy. This reduces sensor network performance when sensor sensitivities and specificities are not the same.

\section{c) Bayesian Confidence Fusion}

An alternative method of combining data from multiple sources is to apply Bayesian techniques to estimate an overall confidence, given the data, whether an event has happened. This can be done using Bayes' rule [19] where $P(E)$ is the probability of the event occurring and $P(V)$ is the probability of getting a particular data set of values from the sensors:

$$
P(E \mid V)=P(V \mid E) \cdot P(E) / P(V)
$$

The data set probability can be obtained using the total probability rule [20], and therefore the confidence can be written as follows, where $P\left(E^{\prime}\right)$ represents the probability of the event not occurring:

$$
P(E \mid V)=(P(V \mid E) \cdot P(E)) /\left(P(V \mid E) \cdot P(E)+P\left(V \mid E^{\prime}\right) \cdot P\left(E^{\prime}\right)\right)
$$

$P(V \mid E)$ is obtained from the sensor sensitivities. It is the probability of all of them getting the results they did at the same time. If they are independent, this is the product of their probabilities. $P(E)$ is a prior for the probability of the event occurring, and knowledge of this is therefore required. One method of obtaining this is to capture the event's frequency over a time period containing multiple instances of the event, the fraction of time that the event is occurring.

For the metronome-controlled EMG example, the user is opening and closing their hand a rate of $0.5 \mathrm{~Hz}$, with a mark space ratio of 1 . Over a single 4 -second period, both the time that the hand is closed (T1) and the time that the hand is open (TO) are equal to 2 seconds. Therefore, the probability of the event occurring (i.e. the hand being closed), $P(E)$, at any given time is given as follows:

$$
P(E)=T 1 /(T 1+T 0)=2 /(2+2)=0.5
$$

$P\left(V \mid E^{\prime}\right)$, similar to $P(V \mid E)$, is obtained from the sensor specificities, and $P\left(E^{\prime}\right)$ is simply given by:

$$
P\left(E^{\prime}\right)=1-P(E)
$$

All the information needed to perform this calculation can be obtained with the use of calibration data - a section of experimental data acquired before the main experiment where the user performs the same task, where the ground truth is known - and therefore this technique can be used.

The output of the system, Vout, is governed by:

$$
V_{\text {out }}=\left\{\begin{array}{rr}
1 & \text { if } P(E \mid V) \geq 0.5 \\
0 & \text { otherwise }
\end{array}\right.
$$

This system has an advantage over the majority voting in that the accuracy of each sensor is linked to the amount that it contributes toward the sensor network output. Sensors which provide no information, with an accuracy of 50\%, are ignored entirely. Sensors with a $100 \%$ accuracy are always used over all other sensors. The system uses established statistical mathematics to calculate the probability the event took place, so always outputs the most likely eventuality, given the data from and relative accuracies of the sensors. If applied correctly, using accurate probabilities, it is the theoretically optimal method for combining sensors, but calibration data is required.

\section{Analysis}

To assess and compare the models, it is important to have a method of gauging their performance. The metrics that will be used are sensitivity (true positive rate) and specificity (true negative rate).

To measure these metrics, a ground truth is required. As previously described, the experiment devised for this paper requires the user to open and close their hand in time with a $0.5 \mathrm{~Hz}$ metronome. This is a simple, easily repeatable action with a very low possibility of significant error. While the opening and closing of the hand will not perfectly align with the metronome due to factors such as inconsistent reaction time, it will still do so to a very near margin.

Additionally, using a larger time window (i.e. the maximum of 2 seconds) will effectively eliminate these effects, allowing the ground truth to be assumed to be a square wave of period 4 seconds. This ground truth must be aligned with the experiment's start, but assuming it is correctly done so, it is now possible to measure sensitivity and specificity.

If $N 1$ is total number of ground truth $1 \mathrm{~s}$ and $N 1^{*}$ the number of correctly measured $1 \mathrm{~s}, N O$ total number of ground truth $0 \mathrm{~s}$ and $\mathrm{NO}^{*}$ the number of correctly measured $0 \mathrm{~s}$, then sensitivity $(S S)$ and specificity $(S P)$ are defined by:

$$
\begin{aligned}
& S S=N O * / N O \\
& S P=N 1 * / N 1
\end{aligned}
$$

The mean of the sensitivity and specificity will be obtained and plotted for sensor networks of between $n=1$ and $n=5$ sensors. For each value of $n$, the individual sensor probabilities will be varied (by altering artificial noise intensity) to produce 80 points, showing how sensor network probability varies with individual sensor probability.

The measured probabilities will be compared to the predictions of the models. The mean, absolute mean, maximum, minimum and root mean squared errors (RMSE) for each model will be calculated. For each sample $i$ out of $N$ total samples, $y_{p i}$ represents the prediction and $y_{i}$, the measurement [21].

$$
R M S E=\sqrt{ }\left(\left(\sum_{i=1}^{N}\left(y_{p i}-y_{i}\right)^{2}\right) / N\right)
$$

The experiment will be repeated 10 times, with results represented graphically with scatter plots, to which third-order polynomials will be fitted to show the trend of the results.

\section{RESULTS}

\section{A. Nä̈ve Model}

The naive model prediction was plotted against the measured outcomes (Fig. 2). Table 1 shows the errors between the model outcomes and measured results. 


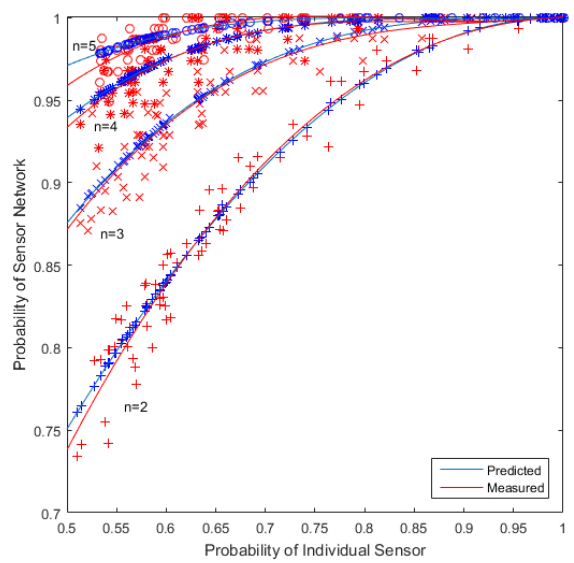

Fig. 2. Plot showing the sensor network probability for $n=2$ to $n=5$ sensors as the mean probability of the individual sensors varies between 0.5 and 1 (controlled by adding artificial noise). Blue points/lines represent the model's prediction. Red points/lines represent the actual measured results. Third-order polynomials have been fitted to demonstrate the trends.

TABLE I. ERROR BETWEEN THE NAÏVE MODEL PREDICTION AND THE MEASURED RESULTS FOR A NAÏVE MODEL FUSION NETWORK OF $N$ SENSORS.

\begin{tabular}{|c|c|c|c|c|c|}
\hline \multirow{2}{*}{ N } & \multirow{2}{*}{ RMSE } & \multicolumn{3}{|c|}{ Absolute Error } & \multirow{2}{*}{ Mean Error } \\
\cline { 3 - 5 } & & Mean & Max & Min & \\
\hline 2 & 0.0142 & 0.0106 & 0.0486 & 0 & -0.0015 \\
\hline 3 & 0.0137 & 0.0100 & 0.0484 & 0 & -0.0015 \\
\hline 4 & 0.0100 & 0.0067 & 0.0315 & 0 & $-8.9748 \mathrm{e}-04$ \\
\hline 5 & 0.0069 & 0.0041 & 0.0314 & 0 & -0.0013 \\
\hline
\end{tabular}

\section{B. Revised Model}

The Revised model prediction was plotted against the measured outcomes (Fig. 3). Table 2 shows the errors between the model outcomes and measured results.

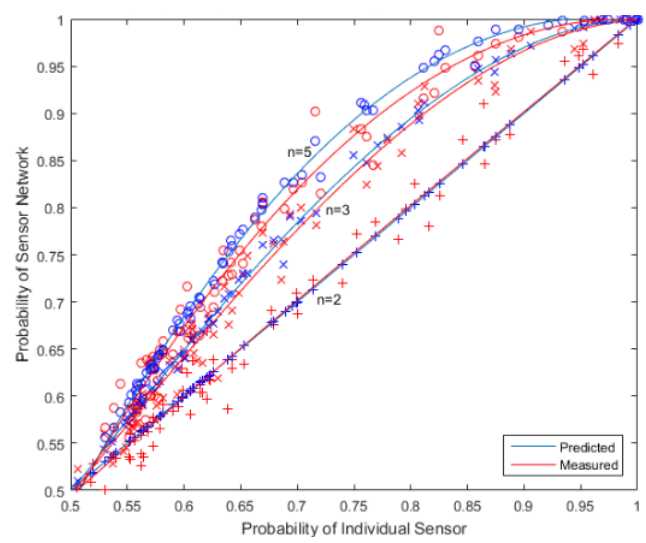

Fig. 3. Plots showing the sensor network probability for $n=2, n=3$ and $n=5$ sensors as the mean probability of the individual sensors varies between 0.5 and 1 (controlled by artificial noise). Blue points/lines represent the model's prediction. Red points/lines represent the actual measured results. Thirdorder polynomials have been fitted to demonstrate the trends of the plot. The $n=4$ results has been omitted, as they overlapped with $n=3$.

TABLE II. ERROR BETWEEN THE REVISED MODEL PREDICTION AND THE MEASURED RESULTS FOR A MAJORITY VOTING NETWORK OF $N$ SENSORS.

\begin{tabular}{|c|c|c|c|c|c|}
\hline \multirow{2}{*}{ N } & \multirow{2}{*}{ RMSE } & \multicolumn{3}{|c|}{ Absolute Error } & \multirow{2}{*}{ Mean Error } \\
\cline { 3 - 5 } & & Mean & Max & Min & \\
\hline 2 & 0.0207 & 0.0150 & 0.0575 & 0 & $-3.4121 \mathrm{e}-04$ \\
\hline 3 & 0.0188 & 0.0141 & 0.0707 & 0 & -0.0053 \\
\hline 4 & 0.0246 & 0.0174 & 0.0761 & 0 & -0.0110 \\
\hline 5 & 0.0207 & 0.0157 & 0.0575 & 0 & -0.0093 \\
\hline
\end{tabular}

\section{Confidence Fusion Method}

Fig. 4 compares the new confidence fusion method with the majority voting method.

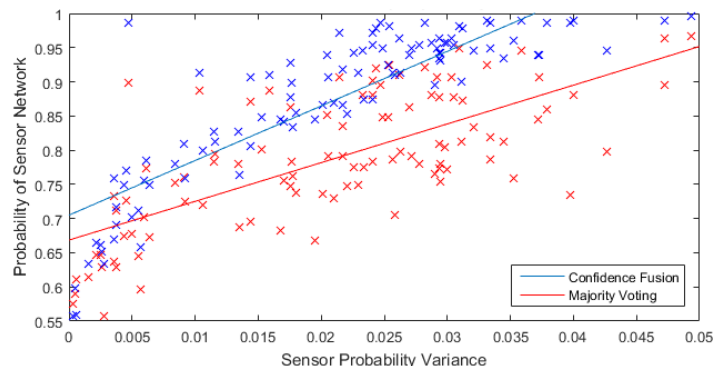

Fig. 4. Graph comparing the new confidence fusion method with the majority voting method for varying selections of sensor probabilities $(n=5)$, plotted according to their variance. Linear best-fit plots have been overlaid in order to show the general trend.

\section{Comparing the Models}

The naïve and revised models were compared to data from majority voting and confidence fusion, averaged over 80 variations of sensor noise intensities (Fig. 5).

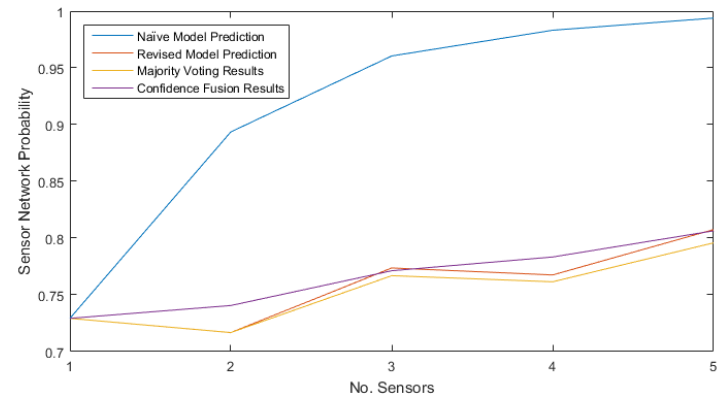

Fig. 5. Graph to show the difference between confidence fusion and majority voting versus the sensor probability variance. A linear best-fit plot has been overlaid to show the general trend.

TABLE III. ERROR BETWEEN THE TWO MODEL PREDICTIONS AND THE MEASURED RESULTS FOR A MAJORITY VOTING NETWORK OF 5 SENSORS.

\begin{tabular}{|l|c|c|c|c|c|}
\hline \multirow{2}{*}{ Model } & \multirow{2}{*}{ RMSE } & \multicolumn{3}{|c|}{ Absolute Error } & \multirow{2}{*}{$\begin{array}{c}\text { Mean } \\
\text { Error }\end{array}$} \\
\cline { 3 - 5 } & & Mean & Max & Min & \\
\hline Naïve & 0.2119 & 0.1580 & 0.4075 & 0 & 0.1580 \\
\hline Revised & 0.0146 & 0.0082 & 0.0527 & 0 & 0.0049 \\
\hline
\end{tabular}

TABLE IV. ERROR BETWEEN THE TWO MODEL PREDICTIONS AND THE MEASURED RESULTS FOR A CONFIDENCE FUSION NETWORK OF 5 SENSORS.

\begin{tabular}{|l|c|c|c|c|c|}
\hline \multirow{2}{*}{ Model } & \multirow{2}{*}{ RMSE } & \multicolumn{3}{|c|}{ Absolute Error } & \multirow{2}{*}{$\begin{array}{c}\text { Mean } \\
\text { Error }\end{array}$} \\
\cline { 3 - 5 } & & Mean & Max & Min & \\
\hline Naïve & 0.1982 & 0.1459 & 0.3986 & 0 & 0.1459 \\
\hline Revised & 0.0226 & 0.0150 & 0.0860 & 0 & -0.0072 \\
\hline
\end{tabular}

\section{DISCUSSION OF RESULTS}

The naïve model shows a good fit with an RMSE of $<1.5 \%$. The model predicts the detection based on EMG data and the measured results are comparable to that of other studies [22]. It should be noted that there was a small negative mean error for all choices of $n$, i.e. the model prediction was a slight overestimate. This kind of error is to be expected due to co-dependency between sensors, as the naïve model assumes that they are independent. 
When predicting the performance of the two practically achievable combination methods (majority voting and confidence fusion) the revised model produced far more accurate predictions than the naïve model. As shown in Tables 3, when analysing EMG data with 80 different combinations of sensor noise in a majority voting system, the revised model gave an RMSE of $\sim 1.5 \%$, whereas that of the naïve model was $\sim 21 \%$. The revised model was slightly less accurate for the confidence fusion method (see Table 4), with an RMSE of $\sim 2.3 \%$, but still far closer to the measured value than the naïve model, which had an RMSE of $\sim 20 \%$.

As expected, the difference between the majority voting and confidence fusion methods is smallest when all sensor probabilities are the same. In this situation, the two methods are identical. As the variance of the sensors increases, the benefit gained from confidence fusion increases, verifying that the confidence fusion method is the preferred sensor fusion method. However, the method can only be used with accurate calibration data, which will need to be repeated whenever the set-up significantly changes. This may impede its practical applicability.

The paper has shown experimentally an application of the naïve model [6]. Descriptive statistics have been generated to compare the model's predictions with real EMG data. These results have shown that, when data can be processed as required, the naïve model is a good estimate. However, implementing it practically is often impossible, as it can require knowledge of the ground truth. The model is, however, useful as an estimate of an upper bound on the best possible performance of a system. While the revised model is a slight overestimate for majority voting and a slight underestimate for confidence fusion, it is a far more effective tool for estimating sensor network performance than the naïve model and is therefore the best available general model to predict the best network configuration for event detection.

\section{A. Recommendations for Future Work}

This paper made extensive use of thresholding in the signal processing stage for reasons of simplicity and ease of mathematical analysis, but more complex alternative methods could be implemented. Future research could focus on this and determine how the choice of processing techniques impacts the optimum number of sensors and environments in a system.

Additionally, the experiments performed used only one subject. While it is expected that similar results would be obtained from repeats using other subjects, EMG signals vary from person to person [23], so a study involving multiple participants from a range of demographics would provide additional verification for the models.

Building upon the foundations established in this paper, it is hoped that future work will push the boundaries of what is achievable through the use of probabilistic sensor networks, accelerating product design with virtual prototyping and using the new models described to bring wearable sensor networks into widespread use, supporting the healthcare industry and thus the wellbeing of the public as a whole.

\section{REFERENCES}

Sensor-Based Systems for Health Monitoring and Prognosis," IEEE Trans. Syst. Man Cybern. - Part C Appl. Rev., vol. 40, no. 1, pp. $1-12,2010$

[2] K. R. Mills, "The Basics of Electromyography," J. Neurol. Neurosurg. Psychiatry, vol. 76, pp. 32-35, 2005.

[3] J. S. Olson and S. Redkar, "A survey of wearable sensor networks in health and entertainment," MOJ Appl. Bionics Biomech., vol. 2, no. 5, pp. 280-287, 2018.

[4] G. G. Wang, "Definition and Review of Virtual Prototyping," $J$. Comput. Inf. Sci. Eng., vol. 2, no. 3, pp. 232-236, 2003.

[5] S. Tilak, N. B. Abu-ghazaleh, and W. Heinzelman, "A Taxonomy of Wireless Micro-Sensor Network Models," ACM SIGMOBILE Mob. Comput. Commun. Rev., vol. 6, no. 2, pp. 28-36, 2002.

[6] J. Bergmann, A. Noble, and M. Thompson, "Probabilistic sensor network design," in 2016 IEEE 13th International Conference on Wearable and Implantable Body Sensor Networks (BSN), 2016, pp. 66-70.

[7] I. A. F. Stokes, S. M. Henry, and R. M. Single, "Surface EMG electrodes do not accurately record from lumbar multifidus muscles," Clin. Biomech. (Bristol, Avon), vol. 18, no. 1, pp. 9-13, 2003

[8] D. A. Winter, A. J. Fuglevand, and S. E. Archer, "Crosstalk in Surface Electromyography: Theoretical and Practical Estimates," J. Electromyogr. Kinesiol., vol. 4, no. 1, pp. 15-26, 1994.

[9] R. Trevethan, "Sensitivity, Specificity, and Predictive Values: Foundations, Pliabilities, and Pitfalls in Research and Practice," Front. Public Heal., vol. 5, p. 307, 2017.

[10] K. Philips and A. van Roermund, Sigma Delta A/D Conversion for Signal Conditioning, 2006 editi. New York: Springer, 2006.

[11] G. B. Giannakis, "Statistical Signal Processing," in Digital Signal Processing Handbook, 1st ed., V. K. Madisetti and D. B. Williams, Eds. Boca Raton: CRC Press, 1999, pp. V-1.

[12] A. P. Dawid, "Conditional Independence in Statistical Theory," $J$. R. Stat. Soc. Ser. B, vol. 41, no. 1, pp. 1-31, 1979.

[13] A. Ragan, "Taking the Confusion Out of Confusion Matrices," Towards Data Science, 2018. [Online]. Available: https://towardsdatascience.com/taking-the-confusion-out-ofconfusion-matrices-c1ce054b3d3e. [Accessed: 01-Jan-2019].

[14] Y. Bai, "Enhancement of the Reliability of an Embedded Surveillance System by Multiple Sensors Using a Majority Voting Mechanism," in 2009 IEEE Instrumentation and Measurement Technology Conference, 2009, no. May, pp. 1157-1162.

[15] PLUX Wireless Biosignals S.A., "BITalino Plugged Kit Data Sheet," 2015. [Online]. Available: http://bitalino.com/datasheets/BITalino_Plugged_Datasheet.pdf. [Accessed: 04-Jan-2019].

[16] SENIAM, "Recommendations for Sensor Locations on Individual Muscles," 2006. [Online]. Available: http://www.seniam.org. [Accessed: 15-Nov-2018].

[17] imusic-school, "Online Metronome," 2018. [Online]. Available: https://www.imusic-school.com/en/tools/online-metronome/. [Accessed: 04-Dec-2018].

[18] C. J. De Luca, "Surface Electromyography: Detection and Recording," Delsys Inc., 2002.

[19] T. Z. Fahidy, "Some Applications of Bayes' Rule in Probability Theory to Electrocatalytic Reaction Engineering," Int. J. Electrochem., vol. 2011, pp. 1-6, 2011.

[20] A. Khrennikov, "Quantum Bayesianism as the basis of general theory of decision-making," Philos. Trans. R. Soc. A Math. Phys. Eng. Sci., vol. 374, 2016.

[21] A. G. Barnston, "Correspondence among the Correlation, RMSE, and Heidke Forecast Verification Measures; Refinement of the Heidke Score," Weather Forecast., vol. 7, no. 4, pp. 699-709, 1992.

[22] K. T. Özgünen, U. Celik, and S. S. Kurdak, "Determination of an Optimal Threshold Value for Muscle Activity Detection in EMG Analysis," J. Sport. Sci. Med., vol. 9, no. 4, pp. 620-628, 2010.

[23] J. Martens, D. Daly, K. Deschamps, R. Jorge, and P. Fernandes, "Intra-Individual Variability of Surface Electromyography in Front Crawl Swimming," PLoS One, vol. 10, no. 12, pp. 1-13, 2015. 